review of clinical guideline implementability. Two analysts independently evaluated each PEM to determine how design principles were applied.

Results Though the sample consisted of PEMs designed and developed to influence care, no single PEM scored well across all categories. Some PEMs failed to differentiate major recommendations and did not present them in a stepwise fashion. Most used clear and easy to read text, but highlighting was often inappropriate. Some algorithms lacked logic and consistency. Images were poorly designed and used, which may distract and confuse the reader.

Discussion Design principles are not consistently applied in the development of PEMs and improvements are needed to images, presentation of recommendations, and usability of algorithms. Improvements to the design of PEMs may influence their uptake by combating information overload and increasing their perceived ease of use and perceived usefulness.

Implications for Guideline Developers/Users Those who create guidelines and other PEMs consider some design principles, but do not implement them consistently. Our checklist can assist guideline developers in employing a range of design principles.

\section{P082 ADHERENCE TO RECOMMENDATIONS OF CLINICAL PRACTICE GUIDELINES}

J Sosa-Garcia, S Martinez-Aldana, D Hernandez-Santillan. National Center for Health Technology Excellence (CENETEC), Mexico

10:1136/bmjqs-2013-002293.151

Background The use of the recommendation of clinical practice guidelines (CPGs) by health professionals, depends on the diffusion process and local strategies of implementation of a particular guide in a specific service of the institution.

Objectives Assess the adherence to the recommendations of CPGs by health professionals internationally.

Methods A systematic review of the literature in PubMed was conducted (MeSH term 'Guideline Adherence', filters: published in the last 5 years, meta-analysis).

Results Out of 33 documents that were obtained, seven were selected, one systematic review and one document in google academic (Mexico). The percentage of adherence differs markedly depending on the directory in question and on the professionals involved from 61.1 to $72.2 \%$. The median adherence was $45 \%$. The professionals with the greatest adherence were dentists, whereas cardiologists and surgeons did not change their behaviour due to the recommendations of a CPG.

Discussion The degree of adherence to the recommendations of the CPG is influenced by different factors, related to the efforts of professional associations, the management of health care organisations, the professionals themselves involved in the care of the patient and the patient himself.

Implications for Guideline Developers/Users The key elements for adherence to the recommendations of the CPG are: involvement of the professionals with the strategy, occupational type, and suggested recommendations.

\section{P084 EXPERIENCES WITH THE NOVEL POLICY FOR MANAGING CONFLICTS OF INTEREST IMPLEMENTED IN THE 9TH EDITION OF THE AMERICAN COLLEGE OF CHEST PHYSICIANS ANTITHROMBOTIC GUIDELINES (AT9)}

1,2 I Neumann, ${ }^{3} \mathrm{R}$ Karl, ${ }^{4} \mathrm{~A}$ Rajpal, $1,5,6 \mathrm{E}$ Akl, ${ }^{1} \mathrm{G}$ Guyatt. ${ }^{1}$ Department of Clinical Epidemiology and Biostatistics, McMaster University, Hamilton, Canada; ${ }^{2}$ Department of
Internal Medicine, Pontificia Universidad Catolica de Chile, Santiago, Chile: ${ }^{3}$ Department of Family Medicine, State University of New York at Buffalo, Buffalo, USA; ${ }^{4}$ Department of Internal Medicine, Drexel School of Medicine, New Jersey, USA; ${ }^{5}$ Department of Internal Medicine, American University of Beirut, Beirut, Lebanon; ${ }^{6}$ Department of Medicine, State University of New York at Buffalo, Buffalo, USA

\section{0:1136/bmjqs-2013-002293.152}

Background The executive committee of the American College of Chest Physicians 9th edition of the Antithrombotic Guidelines (AT9) developed a novel policy for managing conflicts of interest (COI): methodologists bore primary responsibility for each chapter; there was equal emphasis on intellectual and financial COI; and content experts with COI participated, but the intent was to exclude them from the final decisions on recommendations on which they had conflicts.

Objectives To explore the experiences of the AT9 methodologists and content experts with the COI policy.

Methods A descriptive qualitative study: We conducted two rounds of semi-structured interviews with 15 participants and presented the results to the remaining 4 for verification.

Results Methodologists were more positive about the policy than content experts. Six of 10 content experts expressed a more positive view than prior to participation in the AT9 process. The other 4 content experts remained sceptical, especially regarding the emphasis on intellectual COI. It was not possible to completely exclude conflicted panellists from the final decisions of the recommendations on which they had COI.

Discussion After its implementation, some content experts were more favourable to the policy, but some retained major reservations. The influence of the policy on recommendations may have been more through the leading role of the methodologists than exclusion of conflicted participants in making recommendations. Implications for Guideline Developers/Users The leading role of methodologists was a positive innovation. However, restrictions to conflicted panellists were difficult to fully implement.

\section{P086 DESCRIBING GUIDELINE DEVELOPMENT PROCESS: RESPONDING TO NEW CHALLENGES AND ENSURING TRANSPARENCY}

? The Finnish Medical Society Duodecim/Current Care

10:1136/bmjqs-2013-002293.153

Background Evidence-based guidelines should be developed with rigorous methodological standards such as described by AGREE, G-I-N and IOM. One of the main aims is that the development process is repeatable and transparent. To follow these principles, process descriptions and methodological handbook are needed to enable appraisal.

Context Our organisation has developed EBM guidelines for two decades. A methodological handbook was first published in 1998, with the latest (6th) revision published in 2012. Until 2012, processes have been described as simple flowcharts, covering mainly the work phases, not the whole process.

Description of Best Practice Handbook was revised in co-operation with other national EBM organisations. It describes composition of guideline development group, methods for developing a guideline, consensus methods and decision-making process, patient involvement, peer review methods, and updating procedure. The process description was initiated in a workshop where all work phases and activities of guideline development process were written down and placed on a process flow diagram (swimlane) in chronological order. At the same time, the performer for each 